\title{
Scattering theory of cooling and heating in optomechanical systems
}

\author{
André Xuereb, ${ }^{1, *}$ Peter Domokos, ${ }^{2}$ János Asbóth, ${ }^{2,3}$ Peter Horak, ${ }^{4}$ and Tim Freegarde ${ }^{1}$ \\ ${ }^{1}$ School of Physics and Astronomy, University of Southampton, Southampton SO17 1BJ, United Kingdom \\ ${ }^{2}$ Research Institute of Solid State Physics and Optics, Hungarian Academy of Sciences, P.O. Box 49, H-1525 Budapest, Hungary \\ ${ }^{3}$ Leiden Institute of Physics, P.O. Box 9504, NL 2300 RA Leiden, The Netherlands \\ ${ }^{4}$ Optoelectronics Research Centre, University of Southampton, Southampton SO17 1BJ, United Kingdom
}

(Received 18 March 2009; published 6 May 2009)

\begin{abstract}
We present a one-dimensional scattering theory which enables us to describe a wealth of effects arising from the coupling of the motional degree of freedom of scatterers to the electromagnetic field. Multiple scattering to all orders is taken into account. The theory is applied to describe the scheme of a Fabry-Perot resonator with one of its mirrors moving. The friction force, as well as the diffusion, acting on the moving mirror is derived. In the limit of a small reflection coefficient, the same model provides for the description of the mechanical effect of light on an atom moving in front of a mirror.
\end{abstract}

DOI: 10.1103/PhysRevA.79.053810

PACS number(s): 42.50.Wk, 37.10.De, 37.10.Vz, 42.70.Qs

\section{INTRODUCTION}

The use of light forces to manipulate mechanical motion has been extended by now from the translational motion of single atoms $[1,2]$ to the motional modes of massive systems, such as the oscillations of a micromechanical mirror [3-5]. The theoretical approach to describing the mechanical effect of light on the center-of-mass motion of atoms is completely distinct from the models dealing with vibrating optical resonators. In the first case, theories are based on the assumption that atoms are very weak scatterers in free space, negligibly perturbing the impinging bright laser beams [6]. In the other case, the influence of the moving massive component on the radiation field is so strong that it is considered a (moving) boundary condition defining a single or a few modes of the field participating in the optomechanical coupling $[7,8]$. This is clearly the case for a Fabry-Perot-type resonator with one of its mirrors moving [9-11]. We argue that these two cases can be dealt with as two extremes of a general system that can be described in a unified theoretical framework.

In this paper we develop and present a scattering theory for optomechanically coupled systems, allowing for the efficient description of the motion of arbitrary combinations of atoms and mirrors interacting through the radiation field. We will restrict the model to one-dimensional motion and small velocities. The main building block is the beam splitter (BS) transfer matrix [12,13], i.e., the local relation between light field amplitudes at the two sides of a scatterer. We will calculate the radiation force acting on a moving scatterer up to linear order in the velocity. The model is completed by including the quantum fluctuations of the radiation force which stem from the quantized nature of the field. We will determine the momentum-diffusion coefficient corresponding to the minimum quantum noise level.

The system we will consider in some detail is composed of two mirrors; one of them is fixed in space, while the other one is mobile. This is the generic scheme for radiation-

\footnotetext{
*Author to whom correspondence should be addressed. andre.xuereb@soton.ac.uk
}

pressure cooling of moving mirrors [14-16]. At the same time, in the limit of low reflection the moving mirror can equally well represent a single atomic dipole interacting with its mirror image in front of a highly reflecting surface [17-19]. The scattering model description of this example gives a clear recipe for generalizing the method to more complex systems.

\section{MODEL}

Consider a pointlike scatterer, BS, moving along the " $x$ " axis on the trajectory $x_{\mathrm{BS}}(t)$. Outside the scatterer, the electric field $\boldsymbol{E}$ can be expressed in terms of a discrete sum of leftand right-propagating plane-wave modes with different wave numbers $k$ and hence different frequencies $\omega=k c$ :

$$
\boldsymbol{E}=\left\{\begin{array}{c}
\sum_{k}\left[A(k) e^{-i k x-i \omega t}+B(k) e^{i k x-i \omega t}\right]+\text { c.c. } \\
\sum_{k}\left[C(k) e^{-i k x-i \omega t}+D(k) e^{i k x-i \omega t}\right]+\text { c.c. },
\end{array}\right.
$$

where $A(k)$ and $B(k)$ are the mode amplitudes on the left side, with $x<x_{\mathrm{BS}}(t)$, while $C(k)$ and $D(k)$ are the amplitudes on the right side, with $x>x_{\mathrm{BS}}(t)$, of BS. This is a simplifying assumption and all our results also hold for a continuum of field modes. In accordance, the magnetic field is [20]

$$
c \boldsymbol{B}=\left\{\begin{array}{l}
\sum_{k}\left[-A(k) e^{-i k x-i \omega t}+B(k) e^{i k x-i \omega t}\right]+\text { c.c. } \\
\sum_{k}\left[-C(k) e^{-i k x-i \omega t}+D(k) e^{i k x-i \omega t}\right]+\text { c.c. }
\end{array}\right.
$$

As depicted schematically in Fig. 1, the scatterer mixes these waves. Our first goal is the derivation of the transverse ma-

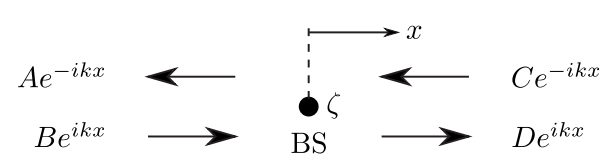

FIG. 1. The four different modes that interact through a pointlike beam splitter in one dimension (1D). 
trix $M$ connecting the field amplitudes on the right side to those on the left side of a beam splitter moving at a fixed velocity $v$. This relation is well known [12] for an immobile scatterer. Therefore, let us first transform the electromagnetic field into a frame moving with the instantaneous velocity $v$ of BS.

\section{A. Transfer matrix for an immobile beam splitter}

In the frame comoving with BS, the interaction of the field with the scatterer at $x^{\prime}=0$ can be characterized by the single parameter $\zeta$ by means of the one-dimensional wave equation $[12,20]$,

$$
\left(\partial_{x^{\prime}}^{2}-\frac{1}{c^{2}} \partial_{t^{\prime}}^{2}\right) \boldsymbol{E}^{\prime}\left(x^{\prime}, t^{\prime}\right)=\frac{2}{k c^{2}} \zeta \delta\left(x^{\prime}\right) \partial_{t^{\prime}}^{2} \boldsymbol{E}^{\prime}\left(x^{\prime}, t^{\prime}\right) .
$$

The electric field can be considered in a modal decomposition similar to Eq. (1). Since a fixed beam splitter couples only the plane waves with identical frequency and wave number, the stationary scattering can be fully described within the closed set of modes

$$
\boldsymbol{E}^{\prime}\left(x^{\prime}, t^{\prime}\right)= \begin{cases}A^{\prime} e^{-i k x^{\prime}-i \omega t^{\prime}}+B^{\prime} e^{i k x^{\prime}-i \omega t^{\prime}}+\text { c.c. }, & x^{\prime}<0 \\ C^{\prime} e^{-i k x^{\prime}-i \omega t^{\prime}}+D^{\prime} e^{i k x^{\prime}-i \omega t^{\prime}}+\text { c.c. }, & x^{\prime}>0,\end{cases}
$$

where the index $k$ has been dropped. A linear relation between the field amplitudes on the right of the scatterer and those on the left can be derived from the wave equation,

$$
\left(\begin{array}{l}
C^{\prime} \\
D^{\prime}
\end{array}\right)=M_{0}\left(\begin{array}{l}
A^{\prime} \\
B^{\prime}
\end{array}\right)
$$

with

$$
M_{0}=\left[\begin{array}{cc}
1-i \zeta & -i \zeta \\
i \zeta & 1+i \zeta
\end{array}\right]=\frac{1}{\mathfrak{t}}\left[\begin{array}{cc}
1 & -\mathfrak{r} \\
\mathfrak{r} & \mathfrak{t}^{2}-\mathfrak{r}^{2}
\end{array}\right] .
$$

In the second form of the transfer matrix $M_{0}$, we expressed it in terms of the reflectivity $\mathfrak{r}$ and transmissivity $\mathfrak{t}$ of the beam splitter. This latter form is more convenient for describing moving mirrors, while for atoms the scattering strength parameter $\zeta$ can be readily expressed in terms of the polarizability [12],

$$
\zeta=\frac{\pi \alpha}{\epsilon_{0} \lambda S}
$$

where $\alpha$ is the linear polarizability and $S$ is the effective beam cross section. For a two-level unsaturated atom with transition frequency $\omega_{\mathrm{A}}$ and linewidth $\Gamma$ [half-width at half maximum (HWHM)], for example,

$$
\zeta=\frac{\sigma_{\mathrm{A}}}{2 S} \frac{\Gamma}{\omega_{\mathrm{A}}-\omega-i \Gamma},
$$

where $\sigma_{\mathrm{A}}=\frac{3 \lambda^{2}}{2 \pi}$ is the resonant radiative cross section of an atom. In this case the transfer matrix depends on the wave number $k$, which might lead to essential effects, e.g., Doppler cooling, close to resonance with the atom (see Sec. II E).

\section{B. Transfer matrix for a moving beam splitter}

The transformation back into the laboratory-fixed frame involves the change of the coordinates, $x^{\prime}=x-v t$ and $t^{\prime}=t$, and the Lorentz boost of the electric field up to linear order in $v / c([20]$, Sec. 11.10):

$$
\boldsymbol{E}=\boldsymbol{E}^{\prime}+v \boldsymbol{B}^{\prime},
$$

where we assumed that $\boldsymbol{E}$ and $\boldsymbol{E}^{\prime}$ are polarized in the " $y$ " direction, $\boldsymbol{B}$ and $\boldsymbol{B}$ ' are polarized in the " $z$ " direction, and the velocity is along the $x$ axis. The electric field in the laboratory frame becomes

$$
\begin{aligned}
\boldsymbol{E}(x, t)= & \sum_{k^{\prime}}\left\{A^{\prime}\left(k^{\prime}\right) e^{-i k^{\prime}(x-v t)-i \omega^{\prime} t}+B^{\prime}\left(k^{\prime}\right) e^{i k^{\prime}(x-v t)-i \omega^{\prime} t}\right. \\
& \left.-\frac{v}{c}\left[A^{\prime}\left(k^{\prime}\right) e^{-i k^{\prime}(x-v t)-i \omega^{\prime} t}-B^{\prime}\left(k^{\prime}\right) e^{i k^{\prime}(x-v t)-i \omega^{\prime} t}\right]\right\} \\
& + \text { c.c. } \\
= & \sum_{k}\left(1-\frac{v}{c}\right) A^{\prime}\left(k+\frac{k v}{c}\right) e^{-i k(1+v / c) x-i \omega t} \\
& +\left(1+\frac{v}{c}\right) B^{\prime}\left(k-\frac{k v}{c}\right) e^{i k(1-v / c) x-i \omega t},
\end{aligned}
$$

which can be expressed as a linear transformation $\hat{L}(v)$ of the amplitudes,

$$
\left(\begin{array}{l}
A(k) \\
B(k)
\end{array}\right)=\hat{L}(-v)\left(\begin{array}{l}
A^{\prime}(k) \\
B^{\prime}(k)
\end{array}\right)
$$

with

$$
\hat{L}(v)=\left[\begin{array}{cc}
\left(1+\frac{v}{c}\right) \hat{P}_{-v} & 0 \\
0 & \left(1-\frac{v}{c}\right) \hat{P}_{v}
\end{array}\right] .
$$

This construction is explored further in Appendix A. Here we defined the operator $\hat{P}_{v}: f(k) \mapsto f\left(k+k \frac{v}{c}\right)$, which represents the Doppler shift of the plane waves in a moving frame. Obviously, $\hat{L}^{-1}(v)=\hat{L}(-v)$ to first order in $\frac{v}{c}$. The total action of the moving BS,

$$
\left(\begin{array}{c}
C(k) \\
D(k)
\end{array}\right)=\hat{M}\left(\begin{array}{c}
A(k) \\
B(k)
\end{array}\right)
$$

can then be obtained from

$$
\hat{M}=\hat{L}(-v) M_{0} \hat{L}(v)=\frac{1}{\mathfrak{t}}\left[\begin{array}{cc}
1 & -\left(1-2 \frac{v}{c}\right) \mathfrak{r} \hat{P}_{2 v} \\
\left(1+2 \frac{v}{c}\right) \mathfrak{r} \hat{P}_{-2 v} & \mathfrak{t}^{2}-\mathfrak{r}^{2}
\end{array}\right],
$$

where we have assumed that $\mathfrak{r}$ and $\mathfrak{t}$ do not depend on the wave number.

Compared to $M_{0}$ in Eq. (4), the difference lies in the off-diagonal terms, including the Doppler shift imposed by 
the reflection on a moving mirror. In other words, the coupled counterpropagating plane-wave modes differ in wave numbers; i.e., $k\left(1+\frac{v}{c}\right)$ right-propagating waves couple to $-k\left(1-\frac{v}{c}\right)$ left-propagating waves. Furthermore, if the polarizability itself depends on the wave number $k$, e.g., as in Eq. (5), the Doppler-shift operator acts also on it. To make this effect explicit, to linear order in $\frac{v}{c}, \hat{M}$ can be written as

$$
\left[\begin{array}{cc}
1-i \zeta-i \frac{v}{c} \omega \frac{\partial \zeta}{\partial \omega} & -i \zeta\left[1-\frac{v}{c}\left(2-\frac{\omega}{\zeta} \frac{\partial \zeta}{\partial \omega}\right)\right] \hat{P}_{2 v} \\
i \zeta\left[1+\frac{v}{c}\left(2-\frac{\omega}{\zeta} \frac{\partial \zeta}{\partial \omega}\right)\right] \hat{P}_{-2 v} & 1+i \zeta-i \frac{v}{c} \omega \frac{\partial \zeta}{\partial \omega}
\end{array}\right] .
$$

The transfer matrix in the laboratory frame can thus be conceived as a $2 \times 2$ supermatrix acting also in the $k$ space. The amplitude $C$ at a given wave number $k$, i.e., $C(k)$, is combined with the amplitudes $A(k)$ and $B\left(k+2 k \frac{v}{c}\right)$. A similar statement holds for $D(k)$.

Starting from the knowledge of the incoming field amplitudes, this transfer matrix allows for calculation of the total electromagnetic field around a beam splitter moving with a fixed velocity. In the next step, we derive the force on the moving scatterer through the Maxwell stress tensor.

\section{Force on a medium in an electromagnetic field}

The Maxwell stress tensor (see [20], Sec. 6.7) is defined, for a homogeneous medium in one dimension $x$, as

$$
\boldsymbol{T}=-\frac{\boldsymbol{\epsilon}_{0}}{2}\left(|\boldsymbol{E}|^{2}+c^{2}|\boldsymbol{B}|^{2}\right),
$$

where the electric field $\boldsymbol{E}$ and the magnetic field $\boldsymbol{B}$ [Eqs. (1) and (2)] have no components along $x$. It is trivial, then, to see that after applying the rotating-wave approximation, we obtain

$$
\boldsymbol{T}_{x x}=-2 \boldsymbol{\epsilon}_{0}\left[\left|\sum_{k} A(k) e^{-i k x-i \omega t}\right|^{2}+\left|\sum_{k} B(k) e^{i k x-i \omega t}\right|^{2}\right],
$$

since the cross terms in $|\boldsymbol{E}|^{2}$ and $|\boldsymbol{B}|^{2}$ have opposite signs. Note that $\boldsymbol{T}$ varies on time scales on the order of the optical period. Let us now introduce a characteristic time $\tau$, over which the variations in $\boldsymbol{T}$ will be averaged. At $x=0$,

$$
\begin{aligned}
\frac{1}{\tau} \int_{0}^{\tau}\left|\sum_{k} A(k) e^{-i \omega t}\right|^{2} d t & =\sum_{k}|A(k)|^{2}+\sum_{i \neq j} \frac{1}{\tau} \int_{0}^{\tau} A\left(k_{i}\right) \\
& \times\left[A\left(k_{j}\right)\right]^{\star} e^{-i\left(\omega_{i}-\omega_{j}\right) t} d t \\
& \approx \sum_{k}|A(k)|^{2}+\sum_{i \neq j} A\left(k_{i}\right)\left[A\left(k_{j}\right)\right]^{\star} \\
& =\left|\sum_{k} A(k)\right|^{2} .
\end{aligned}
$$

In the approximation we assumed that the frequency bandwidth of the excited modes, $\Delta=\max \left\{\omega_{i}-\omega_{j}\right\}$, around the central frequency $\omega_{0}$, is so narrow that $\Delta \tau \ll 2 \pi$. Since the broadening is due to the Doppler shift $\Delta \sim 2 \omega_{0} \frac{v}{c}$, where $v$ is the speed of the beam splitter. For example, taking $v$ to be the typical speed of atoms in a magneto-optical trap, we require $\tau \ll \pi /\left(\omega_{0} \frac{v}{c}\right) \sim 10^{-4}$ s. The time needed to reach the stationary regime of scattering is typically much shorter and thus this condition imposed on the averaging time $\tau$ can be safely fulfilled.

The force on the medium is given by the surface integral of $\boldsymbol{T}$ on the surface, $\mathcal{S}$, of a fictitious volume $V=S \delta l$ enclosing the medium, where $S$ is the mode area and $\delta l$ is the infinitesimal length of the volume along the $x$ axis. Then, this force is given by

$$
\mathbf{F}=\oint_{\mathcal{S}} \boldsymbol{T}_{x x} n_{x} d \mathcal{S}=S\left[\boldsymbol{T}_{x x}\left(x \rightarrow 0^{+}\right)-\boldsymbol{T}_{x x}\left(x \rightarrow 0^{-}\right)\right],
$$

where $n_{x}=\operatorname{sgn}(x)$ is the normal to $\mathcal{S}$. Substituting the relevant expressions for $\boldsymbol{T}$ into the preceding formula gives

$$
\boldsymbol{F}=\frac{\hbar \omega}{c}\left(|A|^{2}+|B|^{2}-|C|^{2}-|D|^{2}\right),
$$

where $A=\left[\hbar \omega / 2 S \epsilon_{0} c\right]^{-1 / 2} \sum_{k} A(k)$ is the photocurrent amplitude, and similarly for $B, C$, and $D$, their modulus square giving the number of photons crossing a unit surface per unit time. Although we considered first the electric field composed of independent modes, in the force expression only the sums of the mode amplitudes occur.

\section{Quantum fluctuations of the force}

In the previous subsection the force was derived based on the assumption that the field amplitudes are $c$ numbers. In order to describe the inherent quantum fluctuations of the force, we need to resort to the quantum theory of fields and represent the mode amplitudes by operators: $A(k) \rightarrow \hat{A}(k)$. To leading order the fluctuations of the force acting on a beam splitter amount to a momentum-diffusion process $[21,22]$. The diffusion coefficient will be evaluated in the following in the minimum quantum noise limit, which occurs in the case of coherent-state fields [23].

The diffusion coefficient can be deduced from the secondorder correlation function of the force operator [24,25],

$$
\left\langle\hat{\boldsymbol{F}}(t) \hat{\boldsymbol{F}}\left(t^{\prime}\right)\right\rangle-\langle\hat{\boldsymbol{F}}(t)\rangle^{2}=\boldsymbol{D}(t) \delta\left(t-t^{\prime}\right) .
$$

The evaluation of this quantum correlation is system specific. Quantum correlations, i.e., the operator algebra of the mode amplitudes $\hat{A}(k), \hat{B}(k), \hat{C}(k)$, and $\hat{D}(k)$, are influenced by multiple scattering and thus depend on the total transfer matrix of the entire system. The simplest case is a single beam splitter where the "input" modes $\hat{B}(k)$ and $\hat{C}(k)$ have independent fluctuations. The calculation, delegated to Appendix $\mathrm{B}$, includes all the steps needed for the treatment of a general system. The diffusion coefficient for a single beam splitter is obtained as

$$
\begin{aligned}
\boldsymbol{D}= & (\hbar k)^{2}\left(|A|^{2}+|B|^{2}+|C|^{2}+|D|^{2}+2 \operatorname{Re}\left\{\mathfrak{r} A^{*} B-\mathfrak{t} A^{*} C\right\}\right. \\
& \left.+2 \operatorname{Re}\left\{\mathfrak{r} D^{*} C-\mathfrak{t} D^{*} B\right\}\right),
\end{aligned}
$$

where $A, B, C, D$ are the photocurrent amplitudes (their modulus square is in the units of $1 / \mathrm{s}$ ), obeying Eq. (6) for $v=0$. 
As an example, let us consider the diffusion coefficient for a two-level atom illuminated by counterpropagating monochromatic light waves. Using the polarizability $\zeta$, the transmission and reflection coefficients can be expressed as $\mathfrak{t}=1 /(1-i \zeta)$ and $\mathfrak{r}=i \zeta /(1-i \zeta)$, respectively [see Eq. (4)]. Equation (11) can then be rewritten in the form

$$
\boldsymbol{D}=(\hbar k)^{2}\left[\frac{2 \operatorname{Im}\{\zeta\}}{|1-i \zeta|^{2}}|B-C|^{2}+\frac{4|\zeta|^{2}}{|1-i \zeta|^{2}}\left(|B|^{2}+|C|^{2}\right)\right]
$$

where the first term, apart from the factor of $|1-i \zeta|^{2}$, corresponds to the result well known from laser-cooling theory, as shown in the next section. Note that the diffusion process due to the recoil accompanying the spontaneous emission of a photon (see [25]) is missing from this result-the detailed modeling of absorption, i.e., scattering photons into the three-dimensional space, is not included in our approach.

\section{E. Example: Force on a moving beam splitter}

We will now use Eq. (9) to derive a general expression for the force on a moving beam splitter illuminated by two counterpropagating monochromatic plane waves with amplitudes $B_{0}$ and $C_{0}$. In using Eq. (6) to express the outgoing field modes in terms of the incoming ones, we note that the outgoing amplitudes comprise two monochromatic terms each:

$$
A=\frac{i \zeta\left[1-\frac{v}{c}\left(2-\frac{\omega}{\zeta} \frac{\partial \zeta}{\partial \omega}\right)\right] B_{0}+C_{0}}{1-i \zeta\left(1+\frac{v}{c} \frac{\omega}{\zeta} \frac{\partial \zeta}{\partial \omega}\right)},
$$

and

$$
D=\frac{\left(1-2 i \frac{v}{c} \omega \frac{\partial \zeta}{\partial \omega}\right) B_{0}+i \zeta\left[1+\frac{v}{c}\left(2-\frac{\omega}{\zeta} \frac{\partial \zeta}{\partial \omega}\right)\right] C_{0}}{1-i \zeta\left(1+\frac{v}{c} \frac{\omega}{\zeta} \frac{\partial \zeta}{\partial \omega}\right)} .
$$

These relations are substituted into Eq. (9), giving

$$
\begin{aligned}
\boldsymbol{F}= & \left\{2 \frac{\hbar \omega}{c} /\left|1-i \zeta\left(1+\frac{v}{c} \frac{\omega}{\zeta} \frac{\partial \zeta}{\partial \omega}\right)\right|^{2}\right\} \\
& \times\left\{\left(\operatorname{Im}\{\zeta\}+|\zeta|^{2}+\frac{1}{2} \frac{v}{c} \omega \frac{\partial|\zeta|^{2}}{\partial \omega}\right)\left(\left|B_{0}\right|^{2}-\left|C_{0}\right|^{2}\right)\right. \\
& -\frac{v}{c}\left(\omega \frac{\partial \operatorname{Im}\{\zeta \zeta}{\partial \omega}-\frac{1}{2} \omega \frac{\partial|\zeta|^{2}}{\partial \omega}+2|\zeta|^{2}\right)\left(\left|B_{0}\right|^{2}+\left|C_{0}\right|^{2}\right) \\
& +2\left(\frac{v}{c} \omega \operatorname{Im}\left\{\zeta^{\star} \frac{\partial \zeta}{\partial \omega}\right\}-\operatorname{Re}\{\zeta\}\right) \operatorname{Im}\left\{B_{0} C_{0}^{\star}\right\} \\
& \left.+2 \frac{v}{c}\left(2 \operatorname{Im}\{\zeta\}-\omega \frac{\partial \operatorname{Im}\{\zeta\}}{\partial \omega}+\frac{1}{2} \omega \frac{\partial|\zeta|^{2}}{\partial \omega}\right) \operatorname{Re}\left\{B_{0} C_{0}^{\star}\right\}\right\} .
\end{aligned}
$$

For $v=0$ this result reduces to the one in [13]. Most of the $v$-dependent terms arise from the frequency dependence of

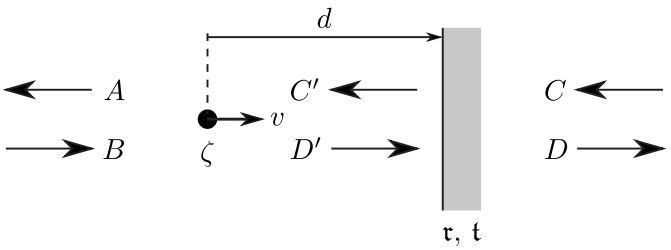

FIG. 2. Physical parameters of our model. $A, B$, etc., represent the field mode amplitudes.

the polarizability. These are the dominant terms in the case of a quasiresonant excitation of a resonant scatterer, such as a two-level atom, since the prefactor $\frac{\omega}{\zeta} \frac{\partial \zeta}{\partial \omega} \sim \frac{\omega}{\Gamma}$ expresses resonant enhancement. The $v$-dependent terms linear in the polarizability $\zeta$ are in perfect agreement with the friction forces known from standard laser-cooling theory, both for propagating waves and for standing waves. For example, assuming identical laser powers from the two sides, giving a standing wave with wave number $k_{0}$, and averaging spatially gives

$$
\boldsymbol{F}=-4 \hbar k_{0}^{2}\left|B_{0}\right|^{2} \operatorname{Im}\left\{\frac{\partial \zeta}{\partial \omega}\right\} v,
$$

for small $|\zeta|$ and to first order in $\frac{v}{c}$, which can be immediately recognized as the friction force in ordinary Doppler cooling [6] when one uses the definition of $\zeta$ in Eq. (5). Finally, by making similar substitutions into Eq. (12), we obtain

$$
\boldsymbol{D}=8\left(\hbar k_{0}\right)^{2} \operatorname{Im}\{\zeta\}\left|B_{0}\right|^{2} \sin ^{2}\left(k_{0} x\right),
$$

which, excluding the diffusion effects due to spontaneous emission, matches the standard result in [25]. Note, however, that the scattering theory leads to a more general result which is represented by the terms of higher order in $\zeta$. These terms describe the backaction of the scatterer on the field, an effect neglected in free-space laser-cooling theory.

The general result in Eq. (13) reveals that this velocitydependent force also acts on a scatterer whose polarizability is independent of the frequency. This is a very general class and we will only focus on such scatterers in the following.

\section{GENERAL SYSTEM OF A FIXED AND A MOBILE SCATTERER}

Consider the model in Fig. 2, where the scatterer, or "atom," has a polarizability $\zeta$ uniform over the frequency range of interest. Letting $M_{\mathrm{a}}, M_{\mathrm{p}}$, and $M_{\mathrm{m}}$ be the transfer matrices for the atom, propagation, and mirror, respectively, we obtain the relation

$$
\left(\begin{array}{l}
A(k) \\
B(k)
\end{array}\right)=M_{\mathrm{a}} M_{\mathrm{p}} M_{\mathrm{m}}\left(\begin{array}{c}
C(k) \\
D(k)
\end{array}\right),
$$

where 


$$
\begin{aligned}
M_{\mathrm{a}} & =\left[\begin{array}{cc}
1+i \zeta & i \zeta\left(1-2 \frac{v}{c}\right) \hat{P}_{2 v} \\
-i \zeta\left(1+2 \frac{v}{c}\right) \hat{P}_{-2 v} & 1-i \zeta
\end{array}\right] \\
& =\left[\begin{array}{cc}
M_{11} & M_{12} \hat{P}_{2 v} \\
M_{21} \hat{P}_{-2 v} & M_{22}
\end{array}\right], \\
M_{\mathrm{p}} & =\left[\begin{array}{cc}
e^{i k d} & 0 \\
0 & e^{-i k d}
\end{array}\right], \quad M_{\mathrm{m}}=\frac{1}{\mathfrak{t}}\left[\begin{array}{cc}
\mathfrak{t}^{2}-\mathfrak{r}^{2} & \mathfrak{r} \\
-\mathfrak{r} & 1
\end{array}\right] .
\end{aligned}
$$

The distance between the atom and the mirror is denoted by $d$. Note that the free-propagation transfer matrix $M_{\mathrm{p}}$ is nonuniform in the $k$ space, and therefore the Doppler shift has an influence on the phase shift accumulated between two scattering events.

The boundary condition is set as follows. Since there is no incoming field from the right, $C(k)=0$ for all $k$. The incoming field from the left is assumed to be monochromatic, $B(k)=\mathcal{B} \delta\left(k-k_{0}\right)$, with $k_{0}$ being the pump wave number. The resulting field comprises modes with wave numbers in a narrow region around $k_{0}$. In the laboratory frame the field mode $A(k)$ interacts with $B\left(k+2 k \frac{v}{c}\right)$ and $C^{\prime}(k)$ through the Doppler shift, and similarly for $D^{\prime}(k)$. From $C(k)=0$ it directly follows that

$$
\begin{aligned}
A(k)= & {\left[\mathfrak{r} M_{11} e^{i k d}+M_{12} \hat{P}_{2 v} e^{-i k d}\right] } \\
& \times\left[\mathfrak{r} M_{21} \hat{P}_{-2 v} e^{i k d}+M_{22} e^{-i k d}\right]^{-1} B(k) \\
= & \frac{1}{M_{22}}\left[\mathfrak{r} M_{11} e^{i k d}+M_{12} \hat{P}_{2 v} e^{-i k d}\right] e^{i k d} \\
& \times \sum_{n=0}^{\infty}\left(-\mathfrak{r} \frac{M_{21}}{M_{22}}\right)^{n} e^{2 i n k d[1-(n+1)(v / c)]} B\left(k-2 n k \frac{v}{c}\right) .
\end{aligned}
$$

We will need the sum of amplitudes, $\mathcal{A}=\int A(k) d k / \mathcal{B}$, defined relative to the incoming amplitude $\mathcal{B}=\int B(k) d k$. Note that $\int \hat{P}_{v} f(k) d k=\int f(k) d k$. Thus, to first order in $\frac{v}{c}$,

$$
\begin{aligned}
\mathcal{A}= & \frac{M_{12}}{M_{22}}+\left(\frac{M_{12}}{M_{22}}-\frac{M_{11}}{M_{21}}\right) \\
& \times \sum_{n=1}^{\infty}\left(-\mathfrak{r} \frac{M_{21}}{M_{22}}\right)^{n}\left[1+2 i n(n-1) k_{0} d \frac{v}{c}\right] e^{2 i n k_{0} d} .
\end{aligned}
$$

It is worth introducing the reference point at a distance $L=2 N \pi / k_{0}$ from the fixed mirror, where the integer $N$ is such that the moving atom's position $x$ is within a wavelength of this reference point. Then the atom-mirror distance can be replaced by $d=L-x$, and $k_{0} L$ drops from all the trigonometric functions. The solution, Eq. (17), has a clear physical meaning, in that the reflected field $\mathcal{A}$ can be decomposed into an interfering sum of fields: the first term is the reflection direct from the atom, whereas the summation is over the electric field undergoing successive atom-mirror round-trips. We can also write the preceding expression in closed form:

$$
\begin{aligned}
\mathcal{A}= & \frac{1}{1-i \zeta}\left\{i \zeta+\mathfrak{r} \frac{e^{-2 i k_{0} x}}{1-i \zeta-\mathfrak{r} i \zeta e^{-2 i k_{0} x}}\right. \\
& -2 i \frac{v}{c} \zeta\left[1-\frac{\mathfrak{r}^{2} e^{-4 i k_{0} x}}{\left(1-i \zeta-\mathfrak{r} i \zeta e^{-2 i k_{0} x}\right)^{2}}\right. \\
& \left.\left.-2 i k_{0}(L-x) \frac{\mathfrak{r}^{2}(1-i \zeta) e^{-4 i k_{0} x}}{\left(1-i \zeta-\mathfrak{r} i \zeta e^{-2 i k_{0} x}\right)^{3}}\right]\right\} .
\end{aligned}
$$

This result is valid for arbitrary $\zeta$. The main virtue of our approach is clearly seen, in that we can smoothly move from $\zeta=0$, which indicates the absence of the mobile scatterer, to $|\zeta| \rightarrow \infty$, which corresponds to a perfectly reflecting mirror, i.e., a moving boundary condition.

Let us outline some of the generic features of the above calculation that would be encountered in a general configuration of scatterers. By using the formal Doppler-shift operators, we benefit from the transfer-matrix method in keeping the description of the system as a whole within $2 \times 2$ matrices. The input-output relation for the total system is always obtained in a form similar to that of Eq. (16). As long as the Doppler broadening is well below the transient time broadening of the system, the calculation of forces and diffusion requires solely the sum of the mode amplitudes. An important point is that the integrated action of the Doppler-shift operator $\hat{P}_{v}$ on monochromatic fields is a shift in $k$ space. Therefore, by interchanging the order of terms and putting the $\hat{P}_{v}$ terms just to the left of the input field amplitudes, they can be eliminated, such as in Eq. (17). Finally, up to first order in $v / c$, the resulting power series, a trace of multiple reflections, can be evaluated in a closed form, as shown in Eq. (18). In conclusion, the illustrated method lends itself for the description of more complex schemes, for example, the cooling of a moving partially reflective mirror in a highfinesse Fabry-Perot resonator [26].

\section{A. Force acting on the mobile scatterer}

To obtain the force on the moving scatterer, we also need to evaluate $C^{\prime}(k)$ and $D^{\prime}(k)$ :

$$
\left(\begin{array}{c}
C^{\prime}(k) \\
D^{\prime}(k)
\end{array}\right)=\left[\begin{array}{cc}
1-i \zeta & -i \zeta\left(1-2 \frac{v}{c}\right) \hat{P}_{2 v} \\
i \zeta\left(1+2 \frac{v}{c}\right) \hat{P}_{2 v}^{-1} & 1+i \zeta
\end{array}\right]\left(\begin{array}{l}
A(k) \\
B(k)
\end{array}\right),
$$

where we applied the inverse of the transfer matrix $M_{\mathrm{a}}$. Next, we make the definitions

$$
\begin{gathered}
\mathrm{A}=|\mathcal{A}|^{2}, \quad \mathrm{~B}=|\mathcal{B}|^{2}, \\
\mathrm{C}=\frac{1}{\mathrm{~B}}\left|\int C^{\prime}(k) d k\right|^{2}, \quad \mathrm{D}=\frac{1}{\mathrm{~B}}\left|\int D^{\prime}(k) d k\right|^{2},
\end{gathered}
$$

and a simple calculation leads to 


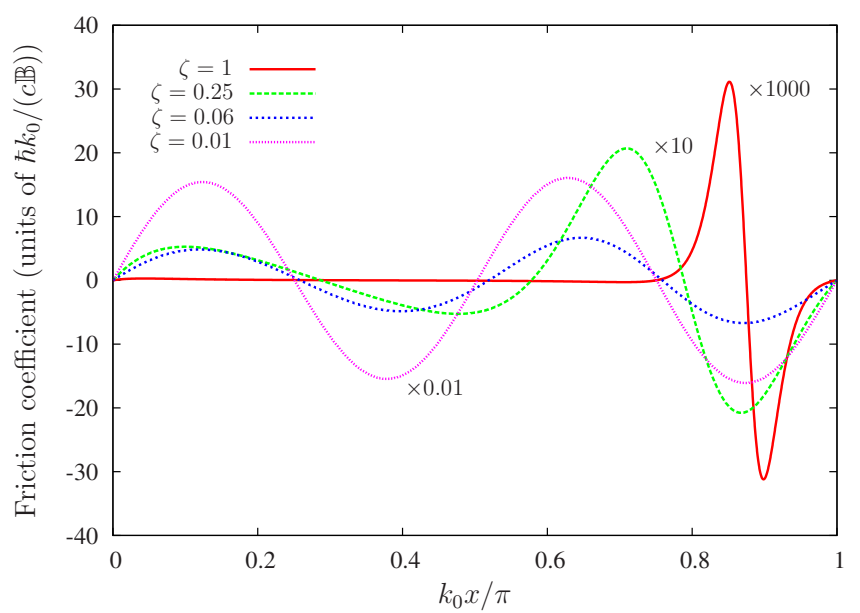

FIG. 3. (Color online) The position dependence of the linear coefficient of the velocity-dependent force acting on the mobile scatterer in Fig. 2, for various scattering parameters $\zeta$, evaluated by using Eqs. (18) and (20) with $k_{0} L=100$. The fixed mirror is assumed to be a perfect mirror. In order to fit all the curves into the same range, they are divided by the factors indicated in the figure.

$$
\begin{aligned}
\mathrm{C}= & |1-i \zeta|^{2} \mathrm{~A}+\left|i \zeta\left(1-2 \frac{v}{c}\right)\right|^{2} \\
& +2 \operatorname{Re}\left\{i \zeta^{\star}(1-i \zeta)\left(1-2 \frac{v}{c}\right) \mathcal{A}\right\}, \\
\mathbb{D}= & \left|i \zeta\left(1+2 \frac{v}{c}\right)\right|^{2} \mathrm{~A}+|1+i \zeta|^{2} \\
& +2 \operatorname{Re}\left\{i \zeta\left(1+i \zeta^{\star}\right)\left(1+2 \frac{v}{c}\right) \mathcal{A}\right\} .
\end{aligned}
$$

Thereby the force acting on the scatterer is obtained as

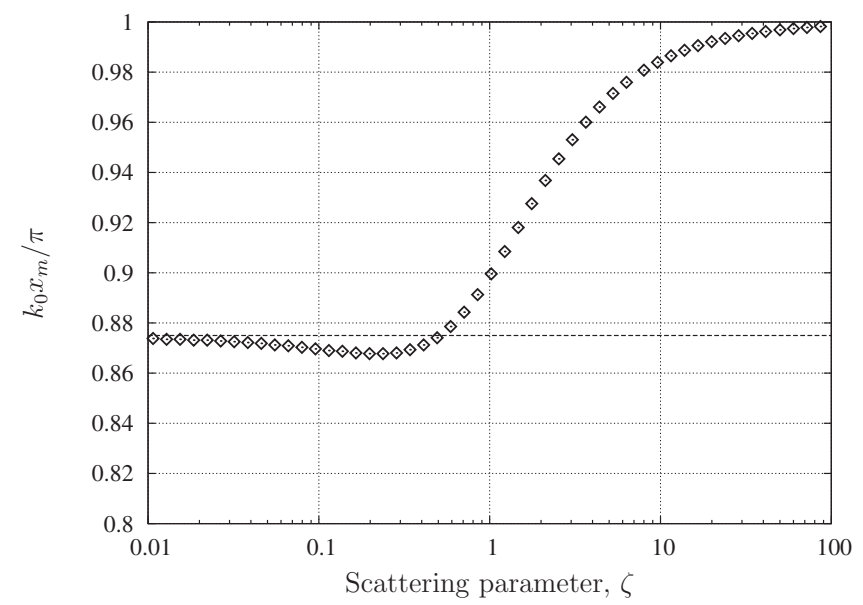

$$
\begin{aligned}
\boldsymbol{F}= & \left(\frac{\hbar \omega}{c}\right) \mathrm{B}(\mathrm{A}+1-\mathrm{C}-\mathbb{D}) \\
= & -2 \hbar k_{0} \mathrm{~B}\left\{\left[|\zeta|^{2}\left(1+2 \frac{v}{c}\right)+\operatorname{Im}\{\zeta\}\right] \mathrm{A}+|\zeta|^{2}\left(1-2 \frac{v}{c}\right)\right. \\
& -\operatorname{Im}\{\zeta\}+2 \operatorname{Re}\{i \zeta(1-i \zeta) \mathcal{A}\}\},
\end{aligned}
$$

where $\mathcal{A}$ has to be substituted from Eq. (18). The coefficient of the term linear in velocity, the "friction coefficient" $\beta$, is plotted in Fig. 3 as a function of the position $x$ in a halfwavelength range for various values of $\zeta$. When varying the coupling strength from $\zeta=0.01$ up to $\zeta=1$, the friction coefficient transforms between two characteristic regimes. For small coupling the linear velocity dependence tends to a simple sinusoidal function, while for large coupling, the friction exhibits a pronounced resonance in a narrow range. This resonance arises from the increased number of reflections between the mobile scatterer and the fixed mirror. It can be observed that the resonance shifts toward $k_{0} x=\pi$ on increasing $\zeta$. In the opposite limit of small $\zeta$, the maximum friction is obtained periodically at $\left(n-\frac{1}{4}\right) \pi / 2$ according to the sinusoidal function. The position of the maximum friction is plotted in Fig. 4(a), showing the transition from $7 \pi / 8$ to $\pi$. The maximum friction force is plotted in Fig. 4(b), showing the two limiting cases of $\zeta^{2}$ behavior, in the limit of small $\zeta$, and $\zeta^{6}$ behavior, in the limit of large $\zeta$. These two cases are described in Secs. IV and V, respectively.

\section{B. Diffusion coefficient}

The calculation of the diffusion coefficient proceeds along the same lines as that corresponding to a single beam splitter, shown in Appendix B. The difference is that the modes $B(k)$ and $C^{\prime}(k)$ around the mobile scatterer are not independent, for the reflection at the fixed mirror mixes them. Therefore, all the modes $A, B, C^{\prime}$, and $D^{\prime}$ have to be expressed in terms of the leftmost and rightmost incoming modes, $B(k)$ and

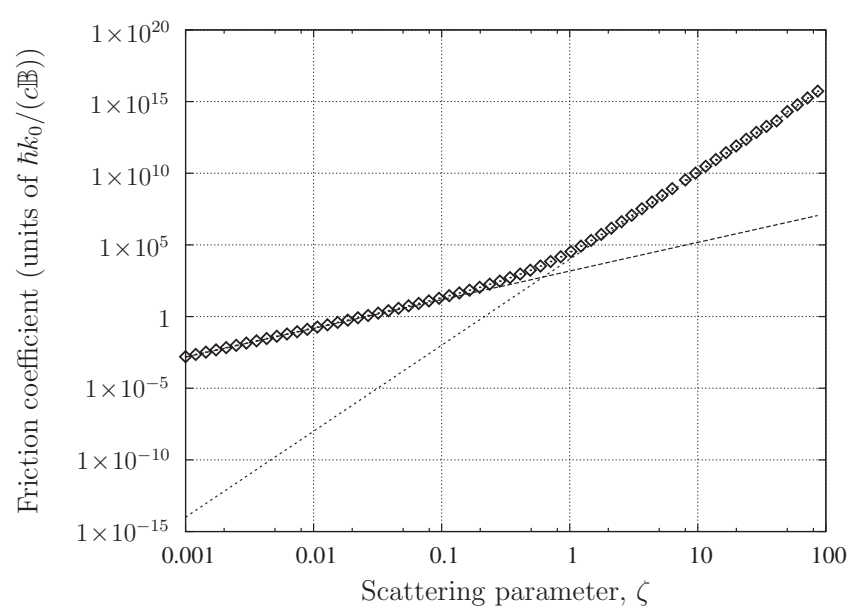

FIG. 4. (a) The position of the maximum friction force $k_{0} x_{m}$ as a function of the dimensionless scattering parameter $\zeta$ (on a semilogarithmic scale) acting on the scatterer in Fig. 2, with the fixed mirror being a perfect mirror. This position shifts from $7 \pi / 8$ to $\pi$ on increasing $\zeta$. (b) A similar plot, showing the maximum friction force as a function of $\zeta$ (on a log-log scale) with $k_{0} L=100$. In the limit of small $\zeta$, the force scales as $\zeta^{2}$ [cf. Eq. (25); dashed line], whereas in the limit of large $\zeta$ it scales as $\zeta^{6}$ [cf. Eq. (27); dotted line]. 


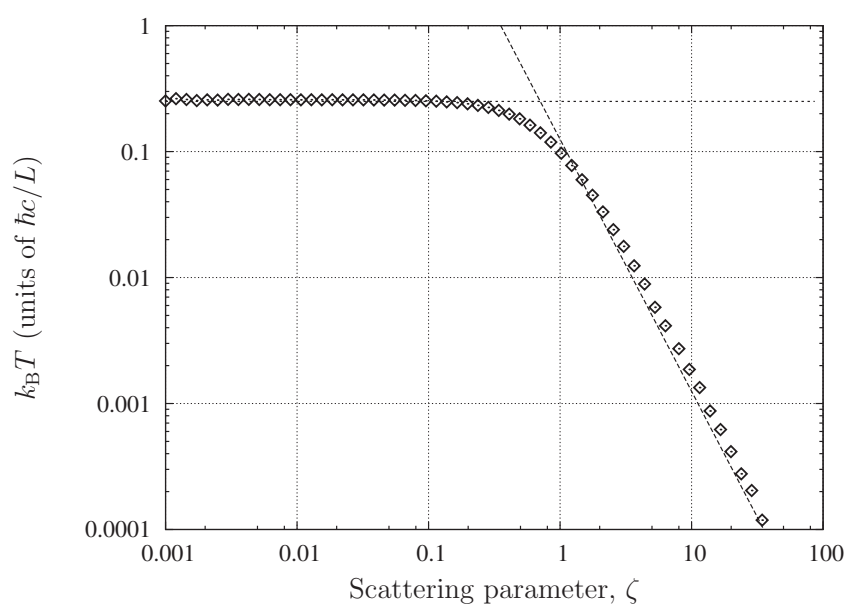

FIG. 5. Characteristic temperature for the two-scatterer system of Fig. 2, given by the ratio of the diffusion and friction coefficients in the points where the friction is maximum, as a function of the dimensionless scattering parameter $\zeta$ on a log-log scale. Constant and $1 / \zeta^{2}$ dependence can be read off in the limits of small and large $\zeta$, respectively. The fixed mirror is a perfect mirror.

$C(k)$, respectively. Instead of the derivation of such a general result for the diffusion, here we will restrict ourselves to the special case of $\mathfrak{r}=-1$ ( $\Leftrightarrow$ perfect mirror) and real $\zeta(\Leftrightarrow$ no absorption in the moving mirror). In this special case the diffusion calculation simplifies a lot, because (i) the perfect mirror prevents the modes $C$ from penetrating into the interaction region, and (ii) quantum noise accompanying absorption does not intrude in the motion of the scatterer.

Only the modes $\hat{B}(k)$ impart independent quantum fluctuations. When all the amplitudes around the scatterer are expressed in terms of $\hat{B}(k)$, and are inserted into the force correlation function given in Eq. (10), the commutator $\left[\hat{b}(t), \hat{b}^{\dagger}\left(t^{\prime}\right)\right]$ appears in all the terms (see Appendix B). Straightforward algebra leads to

$$
\boldsymbol{D}=\hbar^{2} k_{0}^{2} \mathrm{~B}(\mathrm{~A}+1-\mathrm{C}-\mathrm{D})^{2} .
$$

We emphasize that the above result is not general: the diffusion is not necessarily proportional to the square of the force. This simple relation here follows from the assumptions $\mathfrak{r}=$ -1 and $\operatorname{Im}\{\zeta\}=0$ declared above.

To be consistent with the calculation of the friction force linear in velocity, the diffusion should be evaluated only for $v=0$. From the ratio of these two coefficients, the steadystate temperature can be deduced. The velocity-independent components of the modes obey the following relations: $A$ $=1$ and $C^{\prime}=\mathbb{D}^{\prime}$ (all incoming power is reflected). Therefore the diffusion coefficient further simplifies,

$$
\boldsymbol{D}=4 \hbar^{2} k_{0}^{2} \mathbb{B}\left(1-\frac{1}{\left|1-i \zeta+i \zeta e^{-2 i k_{0} x}\right|^{2}}\right)^{2} .
$$

In Fig. 5, the temperature $k_{B} T=\boldsymbol{D} / 2 \beta$, where $\beta$ is the friction coefficient, is plotted as a function of the scattering parameter $\zeta$. The friction and the diffusion coefficients are taken at the position where the friction is maximum, as shown in Fig. 4(a). The two limits of small and large scattering parameter $\zeta$ will be analyzed in Secs. IV and V, respectively.

\section{ATOM IN FRONT OF A PERFECT MIRROR}

An atom pumped with a far off-resonance beam can be modeled as a moving mirror with small and real $\zeta$. In this section we accordingly truncate our expressions to second order in $\zeta$. We also assume that the fixed mirror is perfect; i.e., $\mathfrak{r}=-1$ and $\mathfrak{t}=0$. Thus,

$$
\begin{aligned}
\boldsymbol{F}= & 2 \hbar k_{0} \mathbb{B}\left\{2 \zeta \operatorname{Im}\{\mathcal{A}\}-2 \zeta^{2} \operatorname{Re}\{\mathcal{A}\}\right. \\
& \left.-\zeta^{2}\left(1+\frac{v}{c}\right) A-\zeta^{2}\left(1-\frac{v}{c}\right)\right\} .
\end{aligned}
$$

To obtain $\boldsymbol{F}$ to second order in $\zeta$, we need $\mathcal{A}$ to first order. Using Eqs. (17) and (23), we obtain

$$
\begin{aligned}
\mathcal{A}= & -e^{-2 i k_{0} x}+\zeta\left(i-2 i e^{-2 i k_{0} x}+i e^{-4 i k_{0} x}\right) \\
& +\zeta \frac{v}{c}\left[-2 i+2 i e^{-4 i k_{0} x}-4 k_{0}(L-x) e^{-4 i k_{0} x}\right],
\end{aligned}
$$

and

$$
\begin{aligned}
\boldsymbol{F}= & 4 \hbar k_{0} \mathbb{B}\left(\zeta \sin \left(2 k_{0} x\right)-\zeta^{2}\left\{2 \sin ^{2}\left(k_{0} x\right)\left[4 \cos ^{2}\left(k_{0} x\right)-1\right]\right\}\right. \\
& \left.-\zeta^{2} \frac{v}{c}\left[4 \sin ^{2}\left(2 k_{0} x\right)-4 k_{0}(L-x) \sin \left(4 k_{0} x\right)\right]\right)
\end{aligned}
$$

in agreement with [19]. In the far field $(x \gg \lambda)$, the dominant friction term in the preceding expression is the last term, which renders the $\sin \left(4 k_{0} x\right)$ position dependence shown in Fig. 3 for $\zeta=0.01$.

We are now in a position to derive the diffusion coefficient for this system. By substituting Eq. (24) into Eq. (21) and setting $v=0$, we obtain

$$
\boldsymbol{D}=8\left(\hbar k_{0}\right)^{2} \zeta^{2} \mathrm{~B} .
$$

This allows us to estimate the equilibrium temperature for such a system at a position of maximum friction:

$$
k_{B} T \approx \frac{\hbar}{2 \tau}, \quad \text { where } \tau=\frac{2(L-x)}{c},
$$

which we note is identical in form to the Doppler temperature for a two-level atom undergoing free-space laser cooling [6], but where we have replaced the upper state lifetime, $1 / 2 \Gamma$, by the round-trip time delay between the atom and the mirror. Note that this temperature corresponds to the constant value presented in Fig. 5 for $\zeta<0.1$.

\section{OPTICAL RESONATOR WITH MOBILE MIRROR}

After the small-polarizability case of the previous section, we will now consider the $|\zeta| \rightarrow \infty$ limit. We again assume that the fixed mirror of the resonator is perfect, with $\mathfrak{r}=-1$, and that $C=0$. For simplicity, we assume that the moving mirror has a real polarizability. We expand the field mode ampli- 
tudes as power series in $v / c$, such that $\mathcal{A}=\mathcal{A}_{0}+\frac{v}{c} \mathcal{A}_{1}+\cdots$, and similarly for $\mathcal{C}^{\prime}$.

Let us first calculate the field in the resonator for $v=0$. It follows from Eq. (19) that

$$
\mathcal{C}_{0}^{\prime}=(1-i \zeta) \mathcal{A}_{0}-i \zeta=-\frac{e^{-2 i \varphi}}{1-i \zeta+i \zeta e^{-2 i \varphi}},
$$

with $\varphi=k_{0} d$, which has a maximum at $\varphi_{0}$ obeying

$$
\tan \left(2 \varphi_{0}\right)=-\frac{1}{\zeta}
$$

In the limit of $\zeta \rightarrow \infty$, the resonance is Lorentzian,

$$
\mathcal{C}_{0}^{\prime}=-\frac{e^{-2 i \varphi}}{2 i(1-i \zeta)\left[\left(\varphi-\varphi_{0}\right)-i \frac{1}{4 \zeta^{2}}\right]},
$$

with a width of $1 / 4 \zeta^{2}$

The perfect mirror reflects the total power incoming from the left, $\mathbb{B}$. Moreover, for real $\zeta$, there is no absorption in the moving mirror, so the outgoing intensity has to be equal to the incoming one: $A=1$. This is true if $v=0$; for $v \neq 0$, the field can do work on the mirror. The expansion of the backreflected intensity to linear order in velocity reads $A=1$ $+2 \frac{v}{c} \operatorname{Re}\left\{\mathcal{A}_{0}^{*} \mathcal{A}_{1}\right\}$. Extracting the velocity-dependent terms for the general form of the force in Eq. (20), it reduces to

$$
\boldsymbol{F}_{1}=\frac{v}{c} 4 \hbar k_{0} \mathrm{~B} \zeta \operatorname{Im}\left\{\mathcal{A}_{1} /\left(1+i \zeta-i \zeta e^{2 i \varphi}\right)\right\}
$$

which, after some algebra, leads to

$$
\boldsymbol{F}_{1}=-\frac{1}{2} \frac{v}{c} \hbar k_{0}^{2} L \frac{\left(\varphi-\varphi_{0}\right)}{\zeta^{4}\left[\left(\frac{1}{4 \zeta^{2}}\right)^{2}+\left(\varphi-\varphi_{0}\right)^{2}\right]^{3}} \mathbb{B} .
$$

On substituting $\kappa=c / 4 L \zeta^{2}, \Delta_{C}=-c\left(\varphi-\varphi_{0}\right) / L, \quad \eta^{2} / 2 \kappa=\mathbb{B}$, and $G=c^{2} k_{0}^{2} / L^{2}$, the friction force reduces to that derived from the usual radiation-pressure Hamiltonian in Appendix C.

Expressing the field modes interacting with the mobile mirror in terms of the input field mode and performing a calculation similar to that leading to Eq. (11) readily gives

$$
\boldsymbol{D} \approx 4\left(\hbar k_{0}\right)^{2}\left|\mathcal{C}_{0}^{\prime}\right|^{4} \mathrm{~B} \approx \frac{\left(\hbar k_{0}\right)^{2} \mathrm{~B}}{4 \zeta^{4}\left[\left(\frac{1}{4 \zeta^{2}}\right)^{2}+\left(\varphi-\varphi_{0}\right)^{2}\right]^{2}}
$$

The resulting temperature thereby attains a minimum at $4 \zeta^{2}\left(\varphi-\varphi_{0}\right)=1$, i.e., $\Delta_{C}=-\kappa$, in analogy with free-space Doppler cooling, at which point we have

$$
k_{B} T \approx \frac{\hbar c}{8 \zeta^{2} L}=\frac{1}{2} \hbar \kappa
$$

Again, this asymptotic behavior is reflected in Fig. 5 for large $\zeta$. We note the similarity of the preceding expression with the temperature of an atom cooled in a cavity, in the good cavity limit [27]. We conjecture that this is due to the fact that both systems can be considered to involve the cou- pling of a laser with a system having a decay rate $\kappa$. This result also holds for the case of an atom undergoing mirrormediated cooling, as can be seen in Eq. (26).

It is also important to note that the above discussion only treats the effects of the light fields on the scatterer. As such, the temperature limit [Eq. (28)] is intrinsic to the light forces, and the mechanical damping and heating processes present in a real macroscopic mirror-cooling setup are not taken into account. In practice, these heating processes may dominate over the heating induced by the quantum noise in the light field $[28,29]$. In such cases, radiation-pressure cooling is a possible means to lower the equilibrium temperature owing to the additional optical damping process.

\section{CONCLUSIONS}

We have presented a powerful extension of an existing theoretical framework to analyze the interaction between light and matter. The theory we presented is based on the transfer-matrix method for dealing with the interaction between scatterers and a light field, and is therefore able to handle complex optical systems, made from several elements, with relative ease. Through the use of the Maxwell stress tensor one can calculate the force acting on any of the elements in the system. We have generalized the transfer matrix for slowly moving scatterers. Thereby the corrections first order in $v / c$ can be calculated for the electromagnetic field as well as for the radiation force acting on the scatterer. Furthermore, one can express this force in terms of the operators representing the quantized field modes interacting with the scatterer and consequently derive the momentum diffusion of the scatterer due to the quantum noise present in the fields. Our scattering theory can also transparently cover the whole range of interaction strengths, from the perturbative interaction between a weak standing wave and a single atom to the very strong (quasi-boundary-condition) interaction between a pump light field and a Fabry-Perot cavity with a moving mirror.

We also applied this framework to three different lasercooling configurations: optical molasses, mirror-mediated cooling, and cooling of micromirrors. We derived the forces on an atom arising from its interaction with the light field, as well as an estimate of the equilibrium temperature an ensemble of atoms is expected to reach through this interaction. In the case of optical molasses, which corresponds to the well-known Doppler temperature limit, the theory provides for additional force and diffusion terms related to the effect of the backaction of the atom on the radiation field. Although for single atoms in free space this backaction is feeble, it is responsible for the modification of equilibrium properties $[30,31]$ and for collective effects in large optical lattices [32]. In the latter cases of a moving scatterer in front of a fixed mirror, our results are valid for arbitrary scattering strength, i.e., spanning the parameter range from a single atom to high-reflectivity mirror.

\section{ACKNOWLEDGMENTS}

This work was supported by the UK Engineering and 


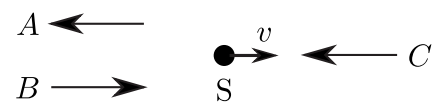

FIG. 6. Reflection and transmission of a moving scatterer. $B$ and $C$ are the input field modes, and $A$ is the output field mode. A further output field mode (" $D$ ") is not drawn because it is not relevant to our discussion in Appendix A.

Physical Sciences Research Council (EPSRC-GB) (Grant No. EP/E058949/1), by the Cavity-Mediated Molecular Cooling working group within the EuroQUAM program of the European Science Foundation (ESF), and by the National Scientific Research Fund of Hungary (Grants No. NF68736 and No. T049234).

\section{APPENDIX A: THE DOPPLER-SHIFT OPERATOR}

Consider the situation in Fig. 6, in the laboratory frame, where $\mathrm{S}$ is a scatterer and suppose that $B$ and $C$ are known. $A(k)$ has contributions arising from both $B\left(k+2 k \frac{v}{c}\right)$ and $C(k)$, where $k$ is any arbitrary wave number, written separately as

$$
A_{B}(k)=a_{1} B\left(k+2 k \frac{v}{c}\right)
$$

and

$$
A_{C}(k)=a_{2} C(k) \text {. }
$$

We can therefore express $A(k)$ as

$$
A(k)=a_{1} B\left(k+2 k \frac{v}{c}\right)+a_{2} C(k) .
$$

Defining $\hat{P}_{v}$ by $\hat{P}_{v}: f(k) \mapsto f\left(k+k \frac{v}{c}\right)$, we have

$$
A(k)=\hat{P}_{2 v} a_{1} B(k)+a_{2} C(k) .
$$

A similar expression, involving $\hat{P}_{v}^{-1}=\hat{P}_{-v}$, holds for $D(k)$. These two operators can then be introduced into Eq. (3) as part of the Lorentz transformation, and thus into the transfer matrix for the moving scatterer, giving rise to the form shown in Eq. (7). The resulting transformation, for the transfer matrix $M$, of a scatterer moving with velocity $v$ can be written as

$$
\left[\begin{array}{cc}
\left(1-\frac{v}{c}\right) \hat{P}_{v} & 0 \\
0 & \left(1+\frac{v}{c}\right) \hat{P}_{v}^{-1}
\end{array}\right] M\left[\begin{array}{cc}
\left(1+\frac{v}{c}\right) \hat{P}_{v}^{-1} & 0 \\
0 & \left(1-\frac{v}{c}\right) \hat{P}_{v}
\end{array}\right],
$$

to first order in $\frac{v}{c}$, where the ordering of the elements of $M$ is as described in the text. Note that this relation is general, in the sense that the elements of $M$ can depend on $k$ (see Sec. II E).

For any finite $v, \hat{P}_{v}$ is trivially a bounded operator, having unit norm. This property follows from the important relation $\int \hat{P}_{v}^{m} f(k) d k=\int f(k) d k$, for any function $f(k)$ and any integer $m$.

This operation can be generalized to $n=2,3$ dimensions. We define a new operator by $\hat{S}_{i}(\mathbf{v}): f(\mathbf{k}) \mapsto f\left(\mathbf{k}+k_{i} \frac{v_{i}}{c} \mathbf{e}_{i}\right)$, where $\mathbf{e}_{i}$ is the unit vector along the $i$ th coordinate axis, $\mathbf{v}$ is the velocity vector of the scatterer, and $\mathbf{x}=\left(x_{1}, x_{2}, \ldots\right)$ for any vector $\mathbf{x}$. In particular, we have $\hat{P}_{v}=\hat{S}_{1}\left(v \mathbf{e}_{1}\right)$. Now, let $\hat{\mathbf{L}}(\mathbf{v})$ be the $2 n \times 2 n$ matrix operator:

$$
\left[\begin{array}{cccc}
\left(1+\frac{v_{1}}{c}\right) \hat{S}_{1}^{-1}(\mathbf{v}) & 0 & 0 & \cdots \\
0 & \left(1-\frac{v_{1}}{c}\right) \hat{S}_{1}(\mathbf{v}) & 0 & \cdots \\
0 & 0 & \left(1+\frac{v_{2}}{c}\right) \hat{S}_{2}^{-1}(\mathbf{v}) & \cdots \\
\vdots & \vdots & \vdots & \ddots
\end{array}\right]
$$

Then, the transfer matrix for the scatterer moving with velocity $\mathbf{v}$ is given by

$$
\hat{\mathbf{L}}(-v) M \hat{\mathbf{L}}(\mathbf{v})
$$

where $M$ is the original transfer matrix for the scatterer, obtained in a manner such as that used to obtain Eq. (4), for example. The ordering of the elements of $M$ is such that it acts on the vector $\left(A_{1}(\mathbf{k}), B_{1}(\mathbf{k}), A_{2}(\mathbf{k}), \ldots\right)$ :

$$
\left(\begin{array}{c}
C_{1}(\mathbf{k}) \\
D_{1}(\mathbf{k}) \\
C_{2}(\mathbf{k}) \\
\vdots
\end{array}\right)=\hat{\mathbf{L}}(-v) M \hat{\mathbf{L}}(\mathbf{v})\left(\begin{array}{c}
A_{1}(\mathbf{k}) \\
B_{1}(\mathbf{k}) \\
A_{2}(\mathbf{k}) \\
\vdots
\end{array}\right)
$$

with $A_{i}(\mathbf{k})$ as the outgoing mode and $B_{i}(\mathbf{k})$ as the incoming mode along the $i$ th axis in the negative half space (assuming that the scatterer is at the origin), and $C_{i}(\mathbf{k})$ as the incoming mode and $D_{i}(\mathbf{k})$ as the outgoing mode in the positive half space.

\section{APPENDIX B: QUANTUM CORRELATION FUNCTION OF THE FORCE OPERATOR}

In quantum theory, we need to replace the mode amplitudes $A(k)$ by operators $\hat{A}(k)$, and similarly for the $B, C$, and $D$ modes. The cross correlation of these operators is not trivial because of the boundary condition connecting the mode amplitudes $A(k), B(k), C(k)$, and $D(k)$. The input modes $\hat{C}(k)$ and $\hat{B}(k)$ can be considered independent, and the commutator is nonvanishing for the creation and annihilation operators of the same mode, e.g.,

$$
\begin{gathered}
{\left[\hat{B}(k), \hat{B}^{\dagger}\left(k^{\prime}\right)\right]=\left[\hat{C}(k), \hat{C}^{\dagger}\left(k^{\prime}\right)\right]=\frac{\hbar \omega}{2 \epsilon_{0} V} \delta_{k, k^{\prime}},} \\
{\left[\hat{B}(k), \hat{C}^{\dagger}\left(k^{\prime}\right)\right]=0,}
\end{gathered}
$$

assuming a discrete mode index of $k$, and a quantization volume $V=S l$ with $S$ as the mode area and $l$ as a fictitious total length of the space in one dimension.

We consider only the $v=0$ case, since our expressions are accurate up to first order in $v / c$. In the quantum description, the linear relation for the output modes is

$$
A(k)=\mathfrak{t} C(k)+\mathfrak{r} B(k)+\sqrt{\gamma} E,
$$




$$
D(k)=\mathfrak{r} C(k)+\mathfrak{t} B(k)+\sqrt{\gamma} E,
$$

where the transmission $\mathfrak{t}=1 / M_{22}=1 /(1-i \zeta)$, and reflection $\mathfrak{r}=M_{12} / M_{22}=i \zeta /(1-i \zeta)$, as above. The fictitious amplitude $E$ represents the quantum noise fed into the system by the absorption. For $\gamma=1-\left(|\mathfrak{r}|^{2}+|\mathfrak{t}|^{2}\right)$, this noise ensures that the output modes obey the same commutation relations as the input ones, namely,

$$
\begin{gathered}
{\left[\hat{A}(k), \hat{A}^{\dagger}\left(k^{\prime}\right)\right]=\left[\hat{D}(k), \hat{D}^{\dagger}\left(k^{\prime}\right)\right]=\frac{\hbar \omega}{2 \epsilon_{0} V} \delta_{k, k^{\prime}},} \\
{\left[\hat{A}(k), \hat{D}^{\dagger}\left(k^{\prime}\right)\right]=0 .}
\end{gathered}
$$

However, the linear dependence implies that commutators between input and output mode operators are

$$
\begin{aligned}
& {\left[\hat{A}(k), \hat{B}^{\dagger}\left(k^{\prime}\right)\right]=\mathfrak{r}\left[\hat{B}(k), \hat{B}^{\dagger}\left(k^{\prime}\right)\right],} \\
& {\left[\hat{A}(k), \hat{C}^{\dagger}\left(k^{\prime}\right)\right]=\mathfrak{t}\left[\hat{C}(k), \hat{C}^{\dagger}\left(k^{\prime}\right)\right],}
\end{aligned}
$$

and similar relations hold for the cross commutators with $D(k)$.

The proper treatment of quantum fluctuations and the derivation of correlation functions require that the explicit time dependence be considered. Let us introduce the timevarying operators

$$
\hat{a}(t)=\sum_{k} \hat{A}(k) e^{-i \omega t},
$$

and similarly for $\hat{b}(t), \hat{c}(t)$, and $\hat{d}(t)$. It follows that

$$
\left[\hat{a}(t), \hat{a}^{\dagger}\left(t^{\prime}\right)\right]=\frac{\hbar \omega}{2 \epsilon_{0} V} \sum_{k} e^{-i \omega\left(t-t^{\prime}\right)} \approx \frac{\hbar \omega}{2 \epsilon_{0} c S} \delta\left(t-t^{\prime}\right) .
$$

Here we used the fact that the nonexcited vacuum modes also contribute to force fluctuations. Therefore the Fouriertype summation extends to a broad frequency range and yields a $\delta\left(t-t^{\prime}\right)$ on the much slower time scale of interest. A similar commutation relation applies to the operators $\hat{b}(t)$, $\hat{c}(t)$, and $\hat{d}(t)$. The cross commutators can be derived directly from those concerning the modes, e.g.,

$$
\left[\hat{a}(t), \hat{b}^{\dagger}\left(t^{\prime}\right)\right]=\mathfrak{r} \frac{\hbar \omega}{2 \epsilon_{0} c S} \delta\left(t-t^{\prime}\right) .
$$

The force operator is

$$
\hat{\boldsymbol{F}}=S\left[\hat{\boldsymbol{T}}_{x x}\left(x \rightarrow 0^{+}\right)-\hat{\boldsymbol{T}}_{x x}\left(x \rightarrow 0^{-}\right)\right],
$$

as before, where

$$
\hat{\boldsymbol{T}}_{x x}(x \rightarrow 0)= \begin{cases}-2 \epsilon_{0}\left[\hat{a}^{\dagger}(t) \hat{a}(t)+\hat{b}^{\dagger}(t) \hat{b}(t)\right], & x \rightarrow 0^{-} \\ -2 \epsilon_{0}\left[\hat{c}^{\dagger}(t) \hat{c}(t)+\hat{d}^{\dagger}(t) \hat{d}(t)\right], & x \rightarrow 0^{+}\end{cases}
$$

is the quantized stress tensor. Assuming that the field is in a coherent state, in all normally ordered products, the mode amplitude operators can be replaced by the corresponding coherent-state amplitudes, which are $c$ numbers: e.g., $\hat{A}(k)$ $\rightarrow A(k)$ and $\hat{A}^{\dagger}(k) \rightarrow A^{\star}(k)$. The force operator in Eq. (B1) is normally ordered in this way. Therefore coherent-state fields render, as a mean value of the quantum expressions, force equation (9) derived from the classical theory based on definition (8). Nontrivial quantum effects arise from nonnormally ordered products, such as the fourth-order product terms of the second-order correlation function of force equation (10). These terms can be evaluated straightforwardly by invoking the above derived commutators to rearrange the product into normal order. As an example, consider

$$
\begin{aligned}
\left\langle\hat{a}^{\dagger}(t) \hat{a}(t) \hat{a}^{\dagger}\left(t^{\prime}\right) \hat{a}\left(t^{\prime}\right)\right\rangle= & \left\langle\hat{a}^{\dagger}(t) \hat{a}^{\dagger}\left(t^{\prime}\right) \hat{a}(t) \hat{a}\left(t^{\prime}\right)\right\rangle \\
& -\left\langle\hat{a}^{\dagger}(t) \hat{a}\left(t^{\prime}\right)\right\rangle \frac{\hbar \omega}{2 \epsilon_{0} c S} \delta\left(t-t^{\prime}\right) .
\end{aligned}
$$

For radiation fields in coherent state, the first term is canceled from the correlation function by the $\left\langle\hat{a}^{\dagger}(t) \hat{a}\left(t^{\prime}\right)\right\rangle^{2}$ term. The coefficient of $\delta\left(t-t^{\prime}\right)$ in the second term is in normal order and can be replaced by $c$ numbers and then calculated identically as the force in Sec. II E,

$$
\left\langle\hat{a}^{\dagger}(t) \hat{a}(t)\right\rangle \approx\left|\sum A(k)\right|^{2}=\frac{\hbar \omega}{2 \epsilon_{0} c S}|A|^{2},
$$

in terms of the photocurrent intensity $|A|^{2}$.

Assembling all similar contributions, originating from the nonvanishing commutators $\left[b, b^{\dagger}\right],\left[c, c^{\dagger}\right],\left[d, d^{\dagger}\right],\left[a, b^{\dagger}\right]$, etc., one obtains Eq. (11), presented in Sec. II D.

\section{APPENDIX C: MIRROR COOLING VIA THE RADIATION- PRESSURE COUPLING HAMILTONIAN}

We describe a generic optomechanical system composed of a single damped-driven field mode coupled to the motion of a massive particle, whose Hamiltonian is given by $[33,34]$

$$
\hat{\mathcal{H}}=\hbar \omega_{c} \hat{a}^{\dagger} \hat{a}+i \hbar \eta\left(\hat{a}^{\dagger} e^{-i \omega t}-\hat{a} e^{i \omega t}\right)+\frac{\hat{p}^{2}}{2 m}+V(\hat{x})+\hbar G \hat{a}^{\dagger} \hat{a} \hat{x},
$$

where $\hat{a}$ and $\hat{a}^{\dagger}$ are the annihilation and creation operators of the mode, and $\hat{x}$ and $\hat{p}$ are the position and momentum operators associated with the motion, and we drop the carets to signify expectation values. The mode is driven by a coherent field with an effective amplitude $\eta$ and frequency $\omega$. This Hamiltonian describes, for example, the radiation-pressure coupling of a moving mirror to the field in a Fabry-Perot resonator. In this case the coupling constant is $G=\omega_{c} / L$, rendering the cavity mode frequency detuning $\omega_{c} x / L$ provided the mirror is shifted by an amount $x$. Since the cavity mode is lossy with a photon escape rate of $2 \kappa$, the total system is dissipative. Thereby, with a proper setting of the parameters, in particular the cavity detuning $\Delta_{C}=\omega-\omega_{C}$, the mirror motion can be cooled. We will determine the corresponding friction force linear in velocity.

In a frame rotating at frequency $\omega$, the Heisenberg equation of motion for the field mode amplitude reads

$$
\dot{\hat{a}}=\left[i\left(\Delta_{C}-G \hat{x}\right)-\kappa\right] \hat{a}+\eta,
$$

where the noise term is omitted. We assume that the mirror moves along the trajectory $x(t) \approx x+v t$ with fixed velocity $v$ during the short time that is needed for the field mode to 
relax to its steady state. The variation in $\hat{a}$ arises from the explicit time dependence and from the motion of the mirror. A steady-state solution is sought in the form of $\hat{a} \approx \hat{a}^{(0)}(x)$ $+v \hat{a}^{(1)}(x)$. On replacing this expansion into the above equation, and using the hydrodynamic derivative $\frac{d}{d t} \rightarrow \frac{\partial}{\partial t}+v \frac{\partial}{\partial x}$, one obtains a hierarchy of equations of different orders of the velocity $v$. To zeroth order the adiabatic field is obtained as

$$
a^{(0)}=\frac{\eta}{-i\left(\Delta_{C}-G x\right)+\kappa} .
$$

The linear response of $a$ to the mirror motion is then

$$
a^{(1)}=\frac{1}{i\left(\Delta_{C}-G x\right)-\kappa} \frac{\partial}{\partial x} a^{(0)}=\frac{i \eta G}{\left[-i\left(\Delta_{C}-G x\right)+\kappa\right]^{3}} .
$$

The force acting on the mirror derives from the defining equation $\dot{\hat{p}}=\frac{i}{\hbar}[\hat{\mathcal{H}}, \hat{p}]=-\hbar G \hat{a}^{\dagger} \hat{a}$. The force linear in velocity is

$$
\boldsymbol{F}_{1}=-2 v \hbar G \operatorname{Re}\left\{a^{(0) \star} a^{(1)}\right\}=4 v \frac{\hbar \eta^{2} G^{2} \kappa \Delta_{C}}{\left[\Delta_{C}^{2}+\kappa^{2}\right]^{3}},
$$

where we used $x=0$ without loss of generality. It can be seen that mirror cooling requires that $\Delta_{C}<0$, i.e., the cavity resonance frequency is above the pump frequency. In this case, for efficient excitation of the field in the resonator, the frequency of the pump photons is up-shifted at the expense of the mirror's kinetic energy. This cooling force has been derived in Sec. V, as a limiting case of the more general scattering theory. To check the perfect agreement between the two results, the quantity corresponding to $\eta$ can be deduced from the total field energy in the resonator for an immobile mirror, which is $\hbar \omega_{C} \hat{a}^{(0) \dagger} \hat{a}^{(0)}$ here.
[1] T. W. Hänsch and A. L. Schawlow, Opt. Commun. 13, 68 (1975).

[2] D. J. Wineland and W. M. Itano, Phys. Rev. A 20, 1521 (1979).

[3] C. H. Metzger and K. Karrai, Nature (London) 432, 1002 (2004).

[4] T. Corbitt, C. Wipf, T. Bodiya, D. Ottaway, D. Sigg, N. Smith, S. Whitcomb, and N. Mavalvala, Phys. Rev. Lett. 99, 160801 (2007).

[5] A. Schliesser, R. Riviere, G. Anetsberger, O. Arcizet, and T. J. Kippenberg, Nat. Phys. 4, 415 (2008).

[6] H. J. Metcalf and P. van der Straten, J. Opt. Soc. Am. B 20, 887 (2003).

[7] V. Braginsky, Phys. Lett. A 293, 228 (2002).

[8] C. K. Law, Phys. Rev. A 51, 2537 (1995).

[9] S. Gigan, H. R. Bohm, M. Paternostro, F. Blaser, G. Langer, J. B. Hertzberg, K. C. Schwab, D. Bauerle, M. Aspelmeyer, and A. Zeilinger, Nature (London) 444, 67 (2006).

[10] O. Arcizet, P. F. Cohadon, T. Briant, M. Pinard, and A. Heidmann, Nature (London) 444, 71 (2006).

[11] D. Kleckner and D. Bouwmeester, Nature (London) 444, 75 (2006).

[12] I. H. Deutsch, R. J. C. Spreeuw, S. L. Rolston, and W. D. Phillips, Phys. Rev. A 52, 1394 (1995).

[13] J. K. Asbóth, H. Ritsch, and P. Domokos, Phys. Rev. A 77, 063424 (2008).

[14] C. Genes, D. Vitali, P. Tombesi, S. Gigan, and M. Aspelmeyer, Phys. Rev. A 77, 033804 (2008).

[15] I. Wilson-Rae, N. Nooshi, W. Zwerger, and T. J. Kippenberg, Phys. Rev. Lett. 99, 093901 (2007).

[16] F. Marquardt, J. P. Chen, A. A. Clerk, and S. M. Girvin, Phys. Rev. Lett. 99, 093902 (2007).

[17] J. Eschner, C. Raab, F. Schmidt-Kaler, and R. Blatt, Nature
(London) 413, 495 (2001).

[18] P. Bushev, A. Wilson, J. Eschner, C. Raab, F. Schmidt-Kaler, C. Becher, and R. Blatt, Phys. Rev. Lett. 92, 223602 (2004).

[19] A. Xuereb, P. Horak, and T. Freegarde, e-print arXiv:0903.2945, Phys. Rev. A (to be published).

[20] J. D. Jackson, Classical Electrodynamics, 3rd ed. (Wiley, New York, 1998).

[21] J. Dalibard and C. Cohen-Tannoudji, J. Opt. Soc. Am. B 6, 2023 (1989).

[22] Y. Castin and K. Molmer, J. Phys. B 23, 4101 (1990).

[23] R. J. Glauber, Phys. Rev. 131, 2766 (1963).

[24] C. Cohen-Tannoudji, in Fundamental Systems in Quantum Optics, edited by J. Dalibard, J. Zinn-Justin, and J. M. Raimond, Les Houches Summer School (North-Holland, Amsterdam, 1992), pp. 1-164.

[25] J. P. Gordon and A. Ashkin, Phys. Rev. A 21, 1606 (1980).

[26] M. Bhattacharya, H. Uys, and P. Meystre, Phys. Rev. A 77, 033819 (2008).

[27] P. Horak and H. Ritsch, Phys. Rev. A 64, 033422 (2001).

[28] P. R. Saulson, Phys. Rev. D 42, 2437 (1990).

[29] P. F. Cohadon, A. Heidmann, and M. Pinard, Phys. Rev. Lett. 83, 3174 (1999).

[30] M. Weidemüller, A. Görlitz, T. W. Hänsch, and A. Hemmerich, Phys. Rev. A 58, 4647 (1998).

[31] J. K. Asbóth and P. Domokos, Phys. Rev. A 76, 057801 (2007).

[32] J. K. Asbóth, H. Ritsch, and P. Domokos, Phys. Rev. Lett. 98 , 203008 (2007).

[33] J. M. Courty, A. Heidmann, and M. Pinard, Eur. Phys. J. D 17, 399 (2001).

[34] D. Vitali, S. Mancini, L. Ribichini, and P. Tombesi, J. Opt. Soc. Am. B 20, 1054 (2003). 